\title{
El uso de inhibidores de la recaptación selectivos de serotonina durante el embarazo aumentaría la chance de parto prematuro
}

\author{
Selective serotonin reuptake inhibitor use during pregnancy would increase the odds of preterm birth
}

\section{Objetivos}

Evaluar el riesgo de parto prematuro ( $<37$ semanas) en casos de exposición a los inhibidores selectivos de la recaptación de serotonina (IRSS) durante el embarazo.

\section{Fuentes de datos}

Se buscó en bases de datos electrónicas (MEDLINE, Scopus, ClinicalTrials.gov, PROSPERO, EMBASE y Cochrane) desde la incepción hasta mayo de 2015. Se usó una combinación de las siguientes palabras: depresión, embarazo, exposición, antidepresivo, IRSS, inhibidores selectivos de la recaptación de serotonina, nacimiento prematuro, pequeño para la edad gestacional y prematurez.

\section{Selección de estudios}

Se incluyeron estudios con grupo control que evaluaran el efecto de exposición intrauterina a los IRSS y los resultados del embarazo. Todas las cohortes y estudios de casos y controles fueron elegibles para ser incluidos si reportaban incidencia de parto prematuro después de cualquier exposición a IRSS y tenían comparaciones con mujeres embarazadas no expuestas. No hubo restricción por idioma ni por localización geográfica.

\section{Extracción de datos}

El riesgo de sesgo de los estudios incluidos se evaluó mediante
Eke AC, y col. BJOG 2016;123:1900-1907

un instrumento para estudios no aleatorizados (MINORS) ${ }^{1}$.

El resultado primario fue la incidencia de parto prematuro y los secundarios peso al nacer e incidencia del síndrome de distress respiratorio. Se realizaron análisis con subgrupos de estudio con grupo control de mujeres con depresión pero sin exposición a los IRSS durante el embarazo.

\section{Principales resultados}

Se incluyeron ocho estudios (1.237.669 mujeres), 93.982 en el grupo de exposición y 1.143.687 en el grupo control. Después de ajustar por los factores de confusión, la incidencia de riesgo de parto prematuro fue significativamente mayor entre las mujeres tratadas con IRSS comparadas con los controles sin IRSS (OR ajustado 1,24, IC95\% 1,09 a 1,41). En el subgrupo, controlado con mujeres con depresión pero sin exposición a los IRSS también se asoció con mayor parto prematuro $(6,8 \%$ vs $5,8 \%$; OR $1,17$, IC $95 \% 1,10$ a 1,25$)$.

\section{Conclusiones}

Las mujeres que recibieron IRSS durante el embarazo tuvieron mayor chance de parto prematuro en comparación al control con y sin depresión.

Fuente de financiamiento: No referida.

\section{Comentario}

Una de las grandes controversias en caso de embarazo y lactancia tiene que ver con el uso o no de medicación psiquiátrica. Sabemos que todos los psicofármacos que han sido estudiados atraviesan la barrera placentaria y que por lo tanto se recomienda en lo posible, evitar su administración durante el primer trimestre (periodo de organogénesis). Sin embargo, alteraciones como la depresión, con prevalencias del $7 \%$ al $19 \%$ en países desarrollados, nos plantea todo un desafío. Una depresión no tratada durante el embarazo puede conducir a una depresión post-parto, enfermedad seria que puede durar varios meses después de dar a luz y puede afectar significativamente la manera en que la madre se vincula con su hijo. La administración de antidepresivos, particularmente el uso de IRSS ha sido asociado a diversas patologías y riesgos fetales. El presente trabajo que incluyó un gran número de casos en su análisis, muestra un riesgo significativamente mayor de parto prematuro asociado al consumo de IRSS, particularmente en casos de uso de drogas como la fluoxetina y paroxetina. Otros trabajos sostienen que si bien hay evidencias tranquilizadoras para el uso de algunos
IRSS, también hacen referencia a los riesgos de la paroxetina y la fluoxetina en fases tempranas del embarazo mientras que habría algunas evidencias tranquilizadoras para el uso de la sertralina. ${ }^{2}$ La decisión de usar medicación antidepresiva durante el embarazo o lactancia debe pesarse contra los riesgos de depresión materna no tratada. El riesgo-beneficio debe ser discutido cuidadosamente con la paciente, la familia y el obstetra, ya que evitar el uso de antidepresivos durante el embarazo y lactancia, no es a menudo una opción.

\section{Conclusiones del comentador}

Es importante tener en cuenta los resultados de las investigaciones en cuanto a los riesgos del uso de IRSS en el embarazo. Sin embargo, los efectos adversos de una depresión no tratada deben ser considerados. Se debería tener en cuenta la posibilidad de medicar con drogas menos nocivas para la salud de la madre y el feto (por ejemplo sertralina), ya que no tratar la patología psiquiátrica de la madre tiene efectos nocivos o adversos en la relación madre hijo y en el desarrollo del niño.

Silvina Foks [ Área de Salud Mental Perinatal, Servicio de Obstetricia del Hospital Italiano de Buenos Aires silvina.foks@hospitalitaliano.org.ar ]

Foks S. El uso de inhibidores de la recaptación selectivos de serotonina durante el embarazo aumentaría la chance de parto prematuro. Evid Act Pract Ambul. 2017;20(2):57. Comentado de: Eke A, y col. Selective serotonin reuptake inhibitor (SSRI) use during pregnancy and risk of preterm birth: a systematic review and meta-analysis. BJOG 2016;123:1900-1907. PMID: 27239775. 